\title{
Eigenset Generalizations of the Eigenvalue Concept
}

\author{
Charles R. Johnson* \\ Institute for Basic Standards, National Bureau of Standards, Washington, D.C. 20234 \\ (July 14, 1977) \\ For an $n-b y-n$ complex matrix $A$ some generalizations of the eigenvalue-eigenvector equation

$$
A x=\lambda x, 0 \neq x \in C^{n}
$$ \\ are investigated. These take the form

$$
A S=\lambda S \text { or } A S \subseteq \lambda S
$$ \\ where $S$ is a subset of $\mathrm{C}^{n}$ about which various assumptions are made. For example, it is shown that there exists a \\ finite set $S \subset C^{n}$, the sum of whose elements is not 0 , such that $A S=\lambda S$, if and only if $\lambda$ is an eigenvalue of $A$ \\ in the usual sense. The requirement that the sum of the elements of $S$ is not 0 should be viewed as a natural \\ analog of the requirement $x \neq 0$ in the classical eigenvalue-eigenvector equation.
}

Key words: Bounded set; convex hull; eigenvalue-eigenvector equation; root of unity.

Let $M_{n}(C)$ denote the set of $n$-by-n complex matrices. For $A \epsilon M_{n}(C)$, the complex number $\lambda$ is said to be an eigenvalue of $A$ if there exists an $x \in C^{n}$ such that

$$
A x=\lambda x \quad \text { and } \quad x \neq 0 .
$$

We denote the set of all eigenvalues of $A$ by $\sigma(A)$, the spectrum of $A$. For $S$ an arbitrary nonempty subset of $\mathrm{C}^{n}$, we define $A S$ and $\alpha S$, for $A \epsilon M_{n}(C)$ and $\alpha \epsilon C$, in the natural ways; that is, $A S=\{A x: x \epsilon S\}$ and $\alpha S=$ $\{\alpha x: x \in S\}$. We may then consider two generalizations of the classical eigenvalue-eigenvector eq (1):

$$
A S=\lambda S
$$

and

$$
A S \subseteq \lambda S,
$$

and ask (analogously to the classical eigenvalue question) "for which complex numbers $\lambda$ do there exist sets $S$ satisfying (2) or (3)." Of course, if we allow $S=\{0\}$, then for any value of $\lambda$ an $S$ would exist satisfying (2) or (3), and, if we allow $S=C^{n}$, then, for non-singular $A$, any $\lambda \neq 0$ satisfies (2) and, for any $A \epsilon M_{n}(C)$, any $\lambda \neq 0$ satisfies (3). Generally speaking, allowing $S$ to be unbounded results in difficulty putting any restrictions on $\lambda$.

EXAMPLE 1: Let $S$ be the eigenspace corresponding to any nonzero eigenvalue (if there is one) of $A \epsilon M_{n}(C)$. Then, since any nonzero scalar multiple of $S$ is identical to $S$, it follows that (2) and (3) hold for any $\lambda \neq 0$.

REMARK: There is a $\{0\} \neq S \subset C^{n}$ such that (2) or (3) holds for $\lambda=0$, if and only if $\mathrm{A}$ is singular. This means that $\lambda=0$ can satisfy (2) or (3) if and only if $0 \epsilon \sigma(A)$, i.e. 0 is an eigenvalue in the usual sense.

ExAmple 2: Let $A=\left(\begin{array}{ll}0 & 1 \\ 1 & 0\end{array}\right), \lambda=1$ and $S=\left\{\left(\begin{array}{l}0 \\ 1\end{array}\right),\left(\begin{array}{l}1 \\ 0\end{array}\right)\right\}$.

Then (2) is satisfied despite the fact that neither of the two elements of $S$ is an eigenvector.

AMS Subject Classification: 15-A18

* Present address: Institute for Physical Science and Technology, Department of Economics, University of Maryland, College Park, Md. 20742. 
In this note we investigate some generalizations of (1) of the type (2) or (3). In each case some restriction regarding 0 is necessary, and these should be viewed as analogs of the second part of (1). (Of course, if $0 \epsilon$ $S$, then $0 \in A S$ and $0 \epsilon \lambda S$. Thus, neither (2) nor (3) is disturbed by addition of 0 , or deletion of 0 if $S$ does not otherwise intersect the null space of $A$.) The classical case (1) may be viewed as a special case of (2) or (3) in which $S$ has just one element. The first natural generalization of the one element case is the case in which $S$ is finite; the next, the case in which $S$ is bounded.

REMARK: The case of $S$ bounded in (3) might be viewed as a generalization of the instance in which $A$ is a column stochastic matrix, $\lambda=1$, and $S$ is the unit simplex (the convex hull of the $n$ coordinate vectors). A matrix is column stochastic if and only if it maps the unit simplex into itself, and, of course, such a matrix has an eigenvalue equal to 1 .

We first consider the case in which $S$ is finite.

Theorem 1: Let $\mathrm{A} \in \mathrm{M}_{\mathrm{n}}(C)$. There exists a finite set $\mathrm{S} \subset \mathrm{C}^{\mathrm{n}}$ satisfying

$$
\equiv \quad \text { AS }=\lambda S
$$

and $\sum_{v \Phi} \mathrm{v} \neq 0$, if and only if $\lambda \epsilon \sigma(\mathrm{A})$.

Proof: If $\lambda \boldsymbol{\epsilon} \boldsymbol{\sigma}(A)$, let $S=\{x\}$ where $x \neq 0$ is an eigenvector of $A$ corresponding to $\lambda$. Then

$A S=\lambda S, \sum_{v \in S} v \neq 0$ and $S \subset C^{n}$ is finite.

On the other hand, suppose $S \subset C^{n}$ is finite and satisfies (2) and that $x \equiv \sum_{v \in S} v \neq 0$. Then

$$
A x=\sum_{v \in S} A v=\sum_{v \in S} \lambda v=\lambda x
$$

which means that $\lambda \in \boldsymbol{\sigma}(A)$.

EXAMPLE 3. The requirement " $\sum_{v \in S} v \neq 0$ " is necessary in theorem 1 .

If $A=\left(\begin{array}{rr}0 & 1 \\ -1 & 0\end{array}\right), \lambda=1$ and $S=\left\{\left(\begin{array}{l}1 \\ 1\end{array}\right),\left(\begin{array}{r}1 \\ -1\end{array}\right),\left(\begin{array}{r}-1 \\ -1\end{array}\right),\left(\begin{array}{r}-1 \\ 1\end{array}\right)\right\}$, then $A S=\lambda S$ without $\lambda \in \sigma(A)=$ $\{ \pm i\}$. The trouble, of course, is that $\left(\begin{array}{l}1 \\ 1\end{array}\right)+\left(\begin{array}{r}1 \\ -1\end{array}\right)+\left(\begin{array}{r}-1 \\ -1\end{array}\right)+\left(\begin{array}{r}-1 \\ 1\end{array}\right)=\left(\begin{array}{l}0 \\ 0\end{array}\right)$, but it should be noted that $\lambda$ $\boldsymbol{\epsilon} \sigma\left(A^{4}\right)$, and that $\lambda=1=(-i) i$ where one factor is a fourth root of one and the other lies in $\sigma(A)$ (cf. Theorem 2 below).

We next consider what happens if the requirement $\sum_{v \in S} v \neq 0$ of theorem 1 is deleted. This broadens the class of $\lambda$ 's which may occur. A complex number $z$ is called a root of unity if there exists a positive integer $k$ such that $z^{k}=1$.

Theorem 2: Let $\mathrm{A} \notin \mathrm{M}_{\mathrm{n}}(\mathrm{V})$. There exists a finite set $0 \notin \mathrm{S} \subset \mathrm{C}^{\mathrm{n}}$ satisfying

$$
A S \subseteq \lambda S
$$

if and only if $\lambda=\mathrm{z} \alpha$ where $\mathrm{z}$ is a root of unity and $\alpha \epsilon \sigma(\mathrm{A})$.

Proof: First suppose $\lambda=z \alpha$ where $\alpha \epsilon \sigma(\mathrm{A})$ and $z^{k}=1$. Then let $S=\left\{x, z x, \cdots, z^{k-1} x\right\}$ where $0 \neq$ $x \in C^{n}$ satisfies $A x=\alpha x$. It follows that $z S=S$ so that $A S=\alpha S=\alpha(z S)=\lambda S$. Thus $0 € S \subset C^{n}$ satisfies (3) as was to be shown.

On the other hand, suppose $0 \notin S C^{n}$ such that $A S \quad \lambda S$ is given. Since $S$ is finite there must exist a sequence of vectors $x_{i_{1}}, \cdots, x_{i_{k}} \in S$ such that

$$
A x_{i_{j}}=\lambda x_{i_{j+1}}, j=1, \cdots, k-1
$$

and $A x_{i_{k}}=\lambda x_{i_{1}}$. It then follows that

$$
A^{k} x_{i_{1}}=\lambda^{k} x_{i_{1}}
$$


and, therefore, that

$$
\lambda^{k}=\alpha^{k}
$$

for some $\alpha \epsilon \sigma(A)$. If $\lambda=0$, the desired consequence follows trivially, and, if not, this means that $z=\frac{\lambda}{\alpha}$ is a $k$ th root of unity. Since $\lambda=z \cdot \alpha$, this completes the proof.

REMARK: If $0 \epsilon S$ is allowed in the context of theorem 2, then any $\lambda$ may be achieved by letting $S=\{0, x\}$ where $x$ (possibly 0 ) is in the null space of $A$. However, if " $0 \notin S$ " is replaced by " $\{0\} \neq S$ " and " $A S \subseteq \lambda S$ " is replaced by " $A S=\lambda S$ " a valid alternative to theorem 2 results.

We call a set $S \subset C^{n}$ bounded if there is some finite number $r$ (depending on $S$ ) such that the Euclidean length of each element of $S$ is less than $r$. We next consider the case of $S$ bounded in (2) or (3). We denote the convex hull of $T \subset C^{n}$ by $C o(T)$ and the closure of $T$ by $\bar{T}$.

Theorem 3: Let $\mathrm{A} \in \mathrm{M}_{\mathrm{n}}(C)$. There exists a bounded set $\mathrm{S} \subset \mathrm{C}^{\mathrm{n}}$ satisfying

\section{$A S \subseteq \lambda S$}

and $0 \notin \overline{\mathrm{Co}(\mathrm{S})}$, if and only if $\lambda \epsilon \sigma(\mathrm{A})$.

Proof: If $\lambda \epsilon \sigma(A)$, then we may choose $S=\{x\}$ where $0 \neq x \in C^{n}$ is an eigenvector of $A$ corresponding to $\lambda$.

On the other hand, suppose a bounded subset $S$ of $C^{n}$ such that $A S \subseteq \lambda S$ and $0 \notin \overline{C o(S)}$ is given. Then it is straighforward to check that $\hat{S}=\overline{C o(S)}$ satisfies $A \hat{S} \subseteq \lambda \hat{S}$. Now, if $\lambda=0$, the desired result is immediate, and if not, $1 / \lambda A$ is a continuous mapping which maps the compact, convex set $\hat{S}$ into itself. Therefore, by the Brouwer fixed point theorem $1 / \lambda A$ has a fixed point $x \in \hat{S}$. Since $0 \notin \hat{S}$, this means that $x$ is an eigenvector of $A$ corresponding to $\lambda$ and completes the proof.

Remark: The assumption $0 \notin \overline{\mathrm{Co}(S)}$ is, of course, crucial in the above argument. If $0 \in \overline{\mathrm{Co}(S)}$, then 0 might well be the only fixed point of $1 / \lambda A$.

If we relax the requirement $0 \notin \overline{\mathrm{Co}(S)}$ the class of allowable $\lambda$ 's is again broadened.

REMARK: If (2) holds it follows in a straightforward way that for any positive integer $t$,

$$
A^{t} S=\lambda^{t} S \text {. }
$$

Similarly, if (3) holds it follows that

$$
A^{t} S \subseteq \lambda^{t} S
$$

Theorem 4: Let A $\epsilon \mathrm{M}_{\mathrm{n}}(\mathrm{C})$. There exists a bounded set $\{0\} \neq \mathrm{S} \subset \mathrm{C}^{\mathrm{n}}$ satisfying

$$
\mathrm{AS} \subseteq \lambda \mathrm{S}
$$

if and only if $|\lambda| \geq m=\min _{\alpha \in \sigma(\mathrm{A})}|\alpha|$.

Proof: If $|\lambda| \geqslant m$, let $x \neq 0$ be an eigenvector corresponding to an eigenvalue $\alpha$ of $A$ for which $|\alpha|=$ $m$ and let $S=\{a x: a \in C,|a| \leqslant 1\}$. Then $S \neq\{0\}$ and $A S=m S$ while $\lambda S=|\lambda| S$ so that (3) holds.

On the other hand, suppose that $\{0\} \neq S \subset C^{n}$ such that $A S \subseteq \lambda S$ is given, and select an element $0 \neq x \in$ $S$. If $\lambda$ were 0 , then it would follow that $A x=0$ and that $0 \epsilon \sigma(A)$ so that $m$ would be 0 . Thus $|\lambda| \geqslant m$ would be satisfied; and therefore we assume from here on that $\lambda \neq 0$. By continuity we may assume without loss of generality that $A$ has a complete set of linearly independent eigenvectors $x_{1}, \cdots, x_{n}$ corresponding respectively to the eigenvalues $\alpha_{1}, \cdots, \alpha_{n}$. Then $x=\sum_{i=1}^{n} a_{i} x_{i}$ for some complex numbers $a_{1}, \cdots, a_{n}$ and $A^{t} x=\sum_{i=1}^{n} a_{i} \alpha_{i}^{t} x_{i}$. Since (3) implies (5), we obtain that $\sum_{i=1}^{n} a_{i}\left(\frac{\alpha_{i}}{\lambda}\right)^{t} x_{i} \in S$ for all positive integers $t$. If $|\lambda|$ were less than $m$, then the boundedness of $S$ would be contradicted so that we conclude that $|\lambda| \geqslant m$, as was to be shown. 
Only slight modifications of the preceding argument yield the following.

Theorem 5: Let $\mathrm{A} \in \mathrm{M}_{\mathrm{n}}(\mathrm{C})$. There exists a bounded set $\mathrm{S} \subset \mathrm{C}^{\mathrm{n}}$ satisfying $0 \notin \overline{\mathrm{S}}$ and

$$
\mathrm{AS} \subseteq \lambda \mathrm{S}
$$

if and only if $|\lambda|=|\alpha|$ for some $\alpha \epsilon \sigma(\mathrm{A})$.

THeorem 6: Let $\mathrm{A} \epsilon \mathrm{M}_{\mathrm{n}}(\mathrm{C})$. There exists a bounded set $\{0\} \neq \mathrm{S} \subset \mathrm{C}^{\mathrm{n}}$ satisfying

$$
A S=\lambda S
$$

if and only if $|\lambda|=|\alpha|$ for some $\alpha \epsilon \sigma$ (A).

REMARK: Analogous to (3) we may also consider

$$
A S \supseteq \lambda S .
$$

Analysis of (6), however, is similar to that of (3) along the lines of this note. In fact for $\lambda \neq 0$ and $A$ nonsingular, (6) holds if and only if (3) holds with $A$ replaced by $A^{-1}$ and $\lambda$ by $\lambda^{-1}$. It is clear that $\lambda=0$ satisfies (6) if and only if the intersection of $S$ and the null space of $A$ is nonempty. Also, in case $S$ is finite and $\lambda \neq 0,(6)$ implies (2).

We close by noting that many questions are suggested by the above observations. For example, what may be said about the geometric and algebraic properties of eigensets $S$ in the senses of (2) and (3)?

The author would like to express his thanks to Dr. Alan Goldman of the National Bureau of Standards for suggesting some of the questions considered herein.

$$
\text { \&) US GOVERNMENT PRINTING OFFICE: 1977-241-306/4 }
$$

